Supporting Information for:

\title{
$\mathrm{Sr}_{2} \mathrm{LuF}_{7}: \mathrm{Yb}^{3+}-\mathrm{Ho}^{3+}-\mathrm{Er}^{3+}$ Upconverting Nanoparticles as \\ Luminescent Thermometers in the First, Second and Third \\ Biological Windows
}

Marcin Runowski, ${ }^{1, *}$ Szymon Goderski, ${ }^{1}$ Dominika Przybylska, ${ }^{1}$ Tomasz Grzyb, ${ }^{1}$ Stefan Lis, Inocencio R. Martín ${ }^{2}$

${ }^{1}$ Adam Mickiewicz University, Faculty of Chemistry, Uniwersytetu Poznańskiego 8, 61-614 Poznań, Poland

${ }^{2}$ Departamento de Física, MALTA Consolider Team, IMN and IUdEA, Universidad de La Laguna, Apdo. Correos 456, E-38200 San Cristóbal de La Laguna, Santa Cruz de Tenerife, Spain

*Corresponding Author: runowski@amu.edu.pl 


\section{Structure and morphology}

The obtained $\mathrm{Sr}_{2} \mathrm{LuF}_{7}: \mathrm{Yb}^{3+}-\mathrm{Ho}^{3+}-\mathrm{Er}^{3+} \mathrm{NPs}$ crystallize in a form of disordered, fluorite-type, nonstoichiometric compound (solid solution), with elemental composition $\mathrm{M}_{0.67} \mathrm{Ln}_{0.33} \mathrm{~F}_{2.33}$ (per unit cell), cubic crystal system and $F m \overline{3} m$ space group.[1] For the readers' convenience, we simply use thorough the whole article the commonly applied formula with integer numbers, i.e. $\mathrm{M}_{2} \mathrm{LnF}_{7}$. It can be clearly seen (Figure S1) that the recorded powder XRD pattern fits well the reference one, corresponding to the similar cubic $\operatorname{Sr}_{0.84} \mathrm{Lu}_{0.16} \mathrm{~F}_{2.16}$ structure, taken from the International Centre for Diffraction Data (ICDD), card no. 01-0820640. The reflexes broadening is typical of small nanocrystals, and additionally indicate on the formation of the nano-sized material.

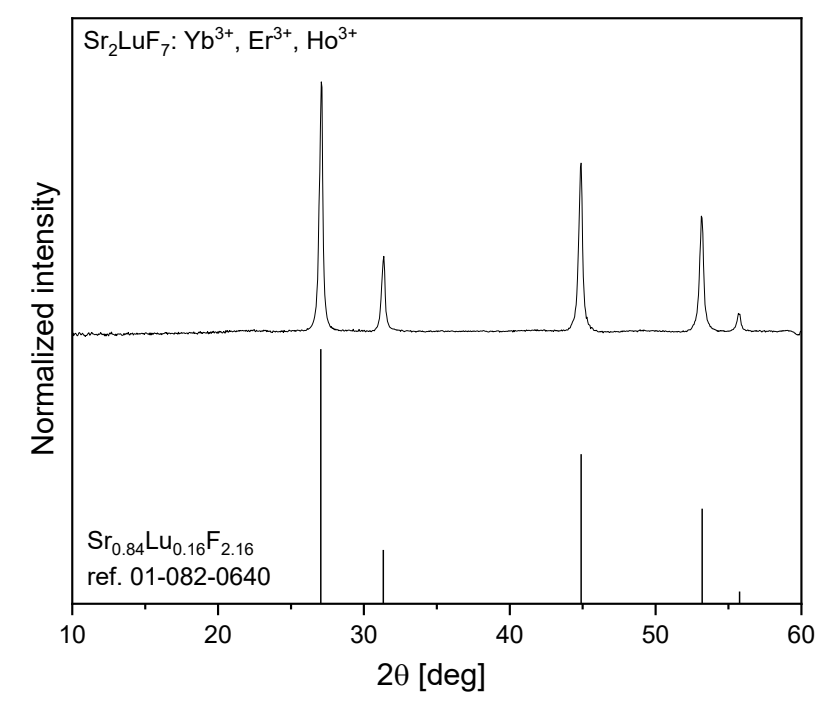

Figure S1. Experimental powder XRD pattern (top) and the reference one (bottom), for the synthesized $\mathrm{Sr}_{2} \mathrm{LuF}_{7}: \mathrm{Yb}^{3+}-\mathrm{Ho}^{3+}-\mathrm{Er}^{3+}$ compound. 
Please note, that the larger $(96 \mathrm{~nm})$ hydrodynamic size of the NPs (Figure S2), compared to TEM-based size $(13 \mathrm{~nm}$; Figure $1 \mathrm{~b})$, is due to the fact that DLS technique provides information about hydrodynamic properties of the compound (including the surface attachment of water molecules, interconnected with a network of hydrogen-bonds). Moreover, the nanoparticles in aqueous environment, usually have tendency to form some agglomerates. That is why, it is typical that the same nanostructures seem to be smaller in TEM images and larger in DLS histogram.

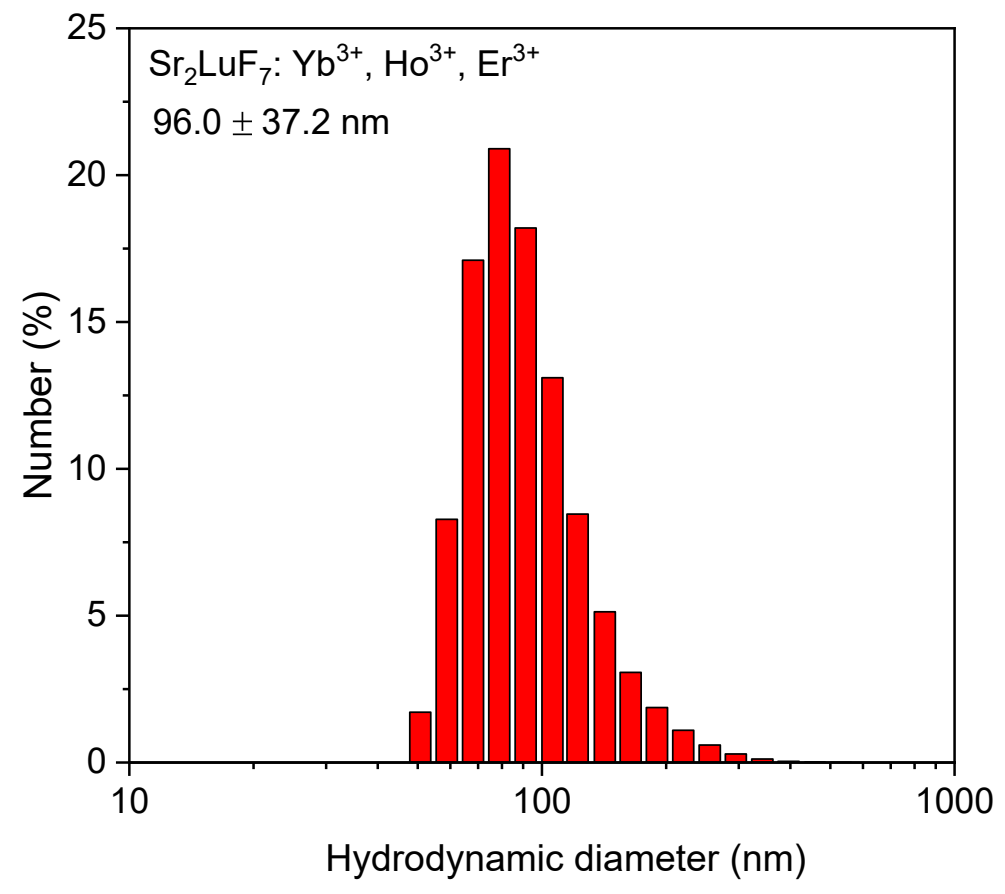

Figure S2. DLS-based (hydrodynamic) size distribution histogram for the obtained $\mathrm{Sr}_{2} \mathrm{LuF}_{7}: \mathrm{Yb}^{3+}-$ $\mathrm{Ho}^{3+}-\mathrm{Er}^{3+} \mathrm{NPs}$. 


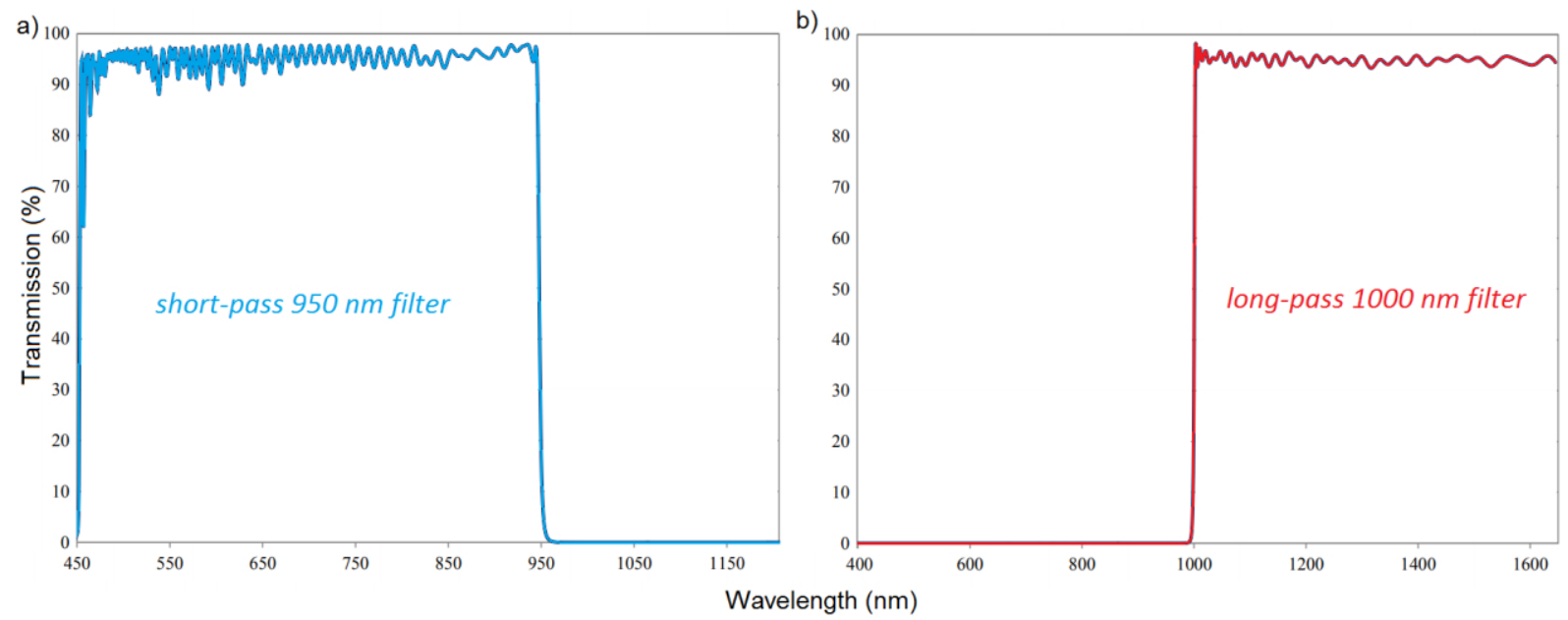

Figure S3. Transmission data for the optical filters used, i.e. (a) short-pass $950 \mathrm{~nm}$ and (b) long-pass $1000 \mathrm{~nm}$ filters.

\section{Luminescence characteristics}

We have used the well-known relation $I_{e m} \propto\left(I_{\text {pump }}\right)^{n}$ to determine the number of photons participating in the transitions corresponding to the observed emission bands of $\mathrm{Ho}^{3+}, \mathrm{Er}^{3+}$ and $\mathrm{Yb}^{3+}$ in the $\mathrm{Sr}_{2} \mathrm{LuF}_{7}: \mathrm{Yb}^{3+}-\mathrm{Ho}^{3+}-\mathrm{Er}^{3+} \mathrm{NPs}$ (see emission spectra in Figures 2 and 4 in the main manuscript). In the mentioned relation, $I_{e m}$ is the emission intensity, $I_{\text {pump }}$ is the laser power and $n$ is the number of photons involved in the given transition. Applying a linear fit, $n$ can be extracted from the slopes of the plotted (in logarithmic scales) emission intensity as a function of the pump power. Please note, that this procedure is commonly used for up-conversion (UC) processes, however, we applied it to all observed emission bands, including $\mathrm{Yb}^{3+}$ and downshifting emission of $\mathrm{Er}^{3+}$ and $\mathrm{Ho}^{3+}$ (above $1000 \mathrm{~nm}$ ), in order to check the potential contribution of the UC processes in the transitions corresponding to those bands.

Figure S4 shows the obtained log-log plots of the integrated luminescence intensities of all observed emission bands in the $\mathrm{Sr}_{2} \mathrm{LuF}_{7}: \mathrm{Yb}^{3+}-\mathrm{Ho}^{3+}-\mathrm{Er}^{3+}$ sample, as a function of pump power. The linear fits were applied in the low excitation power range, mostly from around $\approx 5$ to $\approx 50 \mathrm{~mW}$ (for the three-photon transitions in the blue region, up to $\approx 25 \mathrm{~mW}$ ). At higher pump power values, the initially non-linear (two- or three-photon) UC emission processes 
become more linear, resulting in a decreasing slope values (photon number). This is due to the fact that most of the $\mathrm{Yb}^{3+}$ ions are excited and the saturation processes begin.[2,3] Moreover, at higher laser power the laser-induced sample heating may affect the observed slopes, as well.

The determined slope values are gathered in Table S1. As expected, the slope values for the UC emission bands of $\mathrm{Er}^{3+}$ and $\mathrm{Ho}^{3+}$, located in the spectral range from around 400 to $\approx 515$ are much higher than $2(2.24-2.76)$, whereas the slope values for the UC bands in the range of $\approx 515-870 \mathrm{~nm}$ are much larger than 1 (1.46-1.98), confirming the three- and twophoton UC process, respectively, governing the transitions associated with those bands. Such results are also with good agreement with the presented scheme (Figure 1d), emphasizing the main energy transfer UC processes, occurring in the material studied. The deviations from the ideal values (3 or 2), expected for the pure three- and two-photon processes are related to different non-radiative quenching mechanisms of the excited states of $\mathrm{Er}^{3+}$ and $\mathrm{Ho}^{3+}$, namely multi-phonon relaxation and cross-relaxation processes. For the emission bands located in the range from $\approx 920$ to $1600 \mathrm{~nm}$, the slope values are close to $\approx 1$, which is typical of normal emission bands (non-UC), whose emission intensity is linearly proportional to the applied pump power. The slight differences in the slope value for the same band of $\mathrm{Yb}^{3+}\left({ }^{2} \mathrm{~F}_{5 / 2} \rightarrow{ }^{2} \mathrm{~F}_{7 / 2}\right)$ monitored around $950 \mathrm{~nm}$ (phonon-assisted emission) and above $1000 \mathrm{~nm}$ (down-shifting emission), might be due to some thermal processes (e.g. laser-induced heating) affecting this emission. Whereas, the slightly higher $(>1)$ slope values for the NIR emission bands of $\mathrm{Ho}^{3+}$ $\left({ }^{5} \mathrm{I}_{6} \rightarrow{ }^{5} \mathrm{I}_{8}\right)$ and $\mathrm{Yb}^{3+}\left({ }^{2} \mathrm{~F}_{5 / 2} \rightarrow{ }^{2} \mathrm{~F}_{7 / 2}\right)$, may indicate the contribution of the UC processes (followed by the non-radiative relaxation, and back energy transfer processes, i.e. $\left.\mathrm{Er}^{3+} / \mathrm{Ho}^{3+} \rightarrow \mathrm{Yb}^{3+}\right)$ to the overall intensity of the observed radiative transitions. 

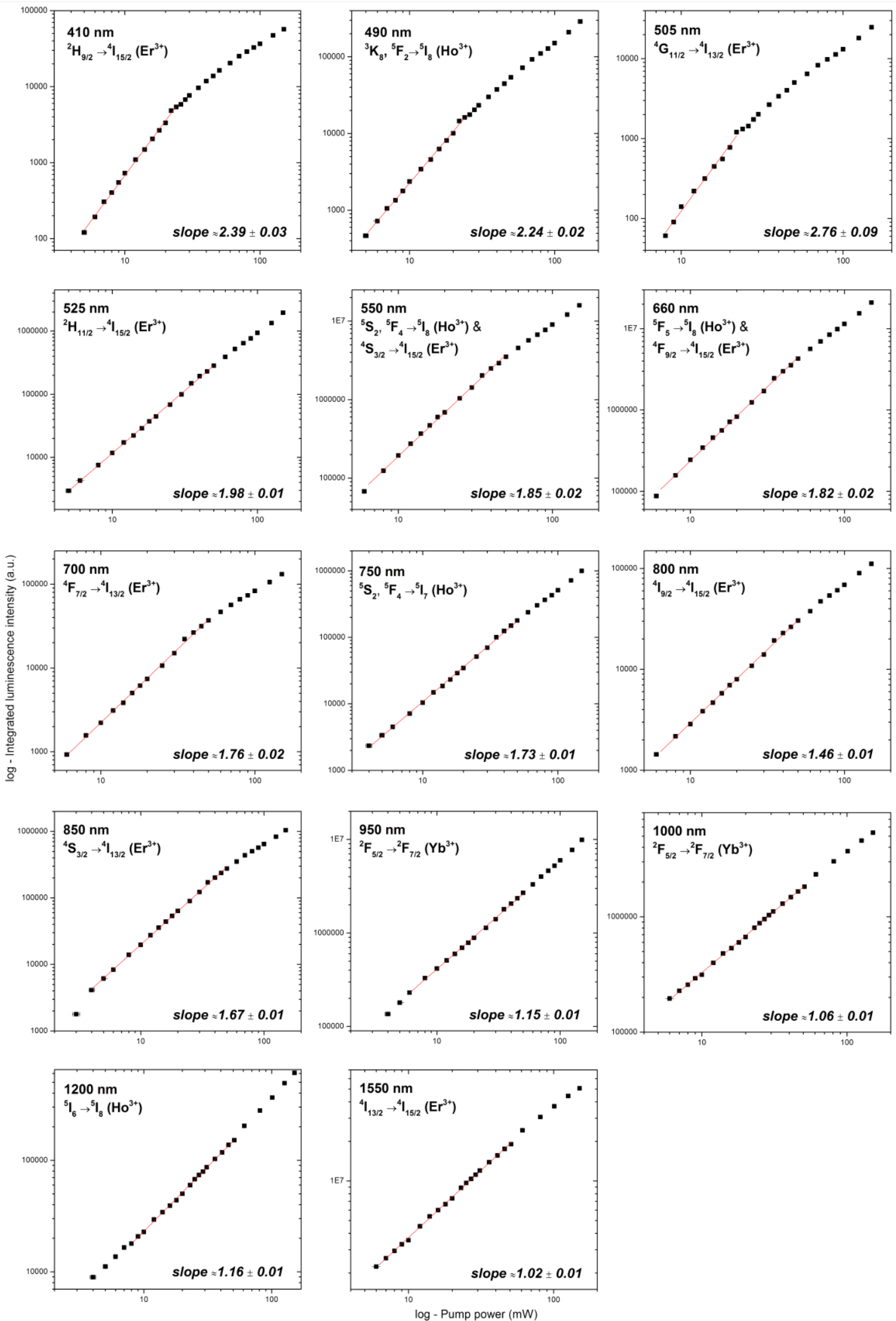

Figure S4. Dependences of the integrated luminescence intensity vs. pump power (log-log plots; both scales in logarithmic representations), for the $\mathrm{Sr}_{2} \mathrm{LuF}_{7}: \mathrm{Yb}^{3+}-\mathrm{Ho}^{3+}-\mathrm{Er}^{3+} \mathrm{NPs}\left(\lambda_{\text {ex }}=975 \mathrm{~nm}\right)$. The 
determined slope values (applying linear fits) correspond to the number of photons participating in the given transition.

Table S1 Determined slope values for the observed radiative transitions, corresponding to the observed emission bands of $\mathrm{Ho}^{3+}, \mathrm{Er}^{3+}$ and $\mathrm{Yb}^{3+}$ in the $\mathrm{Sr}_{2} \mathrm{LuF}_{7}: \mathrm{Yb}^{3+}-\mathrm{Ho}^{3+}-\mathrm{Er}^{3+} \mathrm{NPs}$

\begin{tabular}{ccc}
\hline Transition $\left(\mathrm{Ln}^{3+}\right)$ & Wavelength (nm) & Slope value \\
\hline${ }^{2} \mathrm{H}_{9 / 2} \rightarrow{ }^{4} \mathrm{I}_{15 / 2}\left(\mathrm{Er}^{3+}\right)$ & 410 & $2.39 \pm 0.03$ \\
${ }^{3} \mathrm{~K}_{8},{ }^{5} \mathrm{~F}_{2} \rightarrow{ }^{5} \mathrm{I}_{8}\left(\mathrm{Ho}^{3+}\right)$ & 490 & $2.24 \pm 0.02$ \\
${ }^{4} \mathrm{G}_{11 / 2} \rightarrow{ }^{4} \mathrm{I}_{13 / 2}\left(\mathrm{Er}^{3+}\right)$ & 505 & $2.76 \pm 0.09$ \\
${ }^{2} \mathrm{H}_{11 / 2} \rightarrow{ }^{4} \mathrm{I}_{15 / 2}\left(\mathrm{Er}^{3+}\right)$ & 525 & $1.98 \pm 0.01$ \\
${ }^{5} \mathrm{~S}_{2},{ }^{5} \mathrm{~F}_{4} \rightarrow{ }^{5} \mathrm{I}_{8}\left(\mathrm{Ho}^{3+}\right) \&{ }^{4} \mathrm{~S}_{3 / 2} \rightarrow{ }^{4} \mathrm{I}_{15 / 2}\left(\mathrm{Er}^{3+}\right)$ & 550 & $1.85 \pm 0.02$ \\
${ }^{5} \mathrm{~F}_{5} \rightarrow{ }^{5} \mathrm{I}_{8}\left(\mathrm{Ho}^{3+}\right) \&{ }^{4} \mathrm{~F}_{9 / 2} \rightarrow{ }^{4} \mathrm{I}_{15 / 2}\left(\mathrm{Er}^{3+}\right)$ & 660 & $1.82 \pm 0.02$ \\
${ }^{4} \mathrm{~F}_{7 / 2} \rightarrow{ }^{4} \mathrm{I}_{13 / 2}\left(\mathrm{Er}^{3+}\right)$ & 700 & $1.76 \pm 0.02$ \\
${ }^{5} \mathrm{~S}_{2},{ }^{5} \mathrm{~F}_{4} \rightarrow{ }^{5} \mathrm{I}_{7}\left(\mathrm{Ho}^{3+}\right)$ & 750 & $1.73 \pm 0.01$ \\
${ }^{4} \mathrm{I}_{9 / 2} \rightarrow{ }^{4} \mathrm{I}_{15 / 2}\left(\mathrm{Er}^{3+}\right)$ & 800 & $1.46 \pm 0.01$ \\
${ }^{4} \mathrm{~S}_{3 / 2} \rightarrow{ }^{4} \mathrm{I}_{13 / 2}\left(\mathrm{Er}^{3+}\right)$ & 1550 & $1.67 \pm 0.01$ \\
${ }^{2} \mathrm{~F}_{5 / 2} \rightarrow{ }^{2} \mathrm{~F}_{7 / 2}\left(\mathrm{Yb}^{3+}\right)$ & 850 & $1.15 \pm 0.01$ \\
${ }^{2} \mathrm{~F}_{5 / 2} \rightarrow{ }^{2} \mathrm{~F}_{7 / 2}\left(\mathrm{Yb}^{3+}\right)$ & 950 & $1.06 \pm 0.01$ \\
${ }^{5} \mathrm{I}_{6} \rightarrow{ }^{4} \mathrm{I}_{13 / 2} \rightarrow{ }^{5} \mathrm{I}_{8}\left(\mathrm{Ho}^{3+} \mathrm{I}_{15 / 2}\left(\mathrm{Er}^{3+}\right)\right.$ & 1000 & $1.16 \pm 0.01$ \\
\hline & & \\
\hline
\end{tabular}




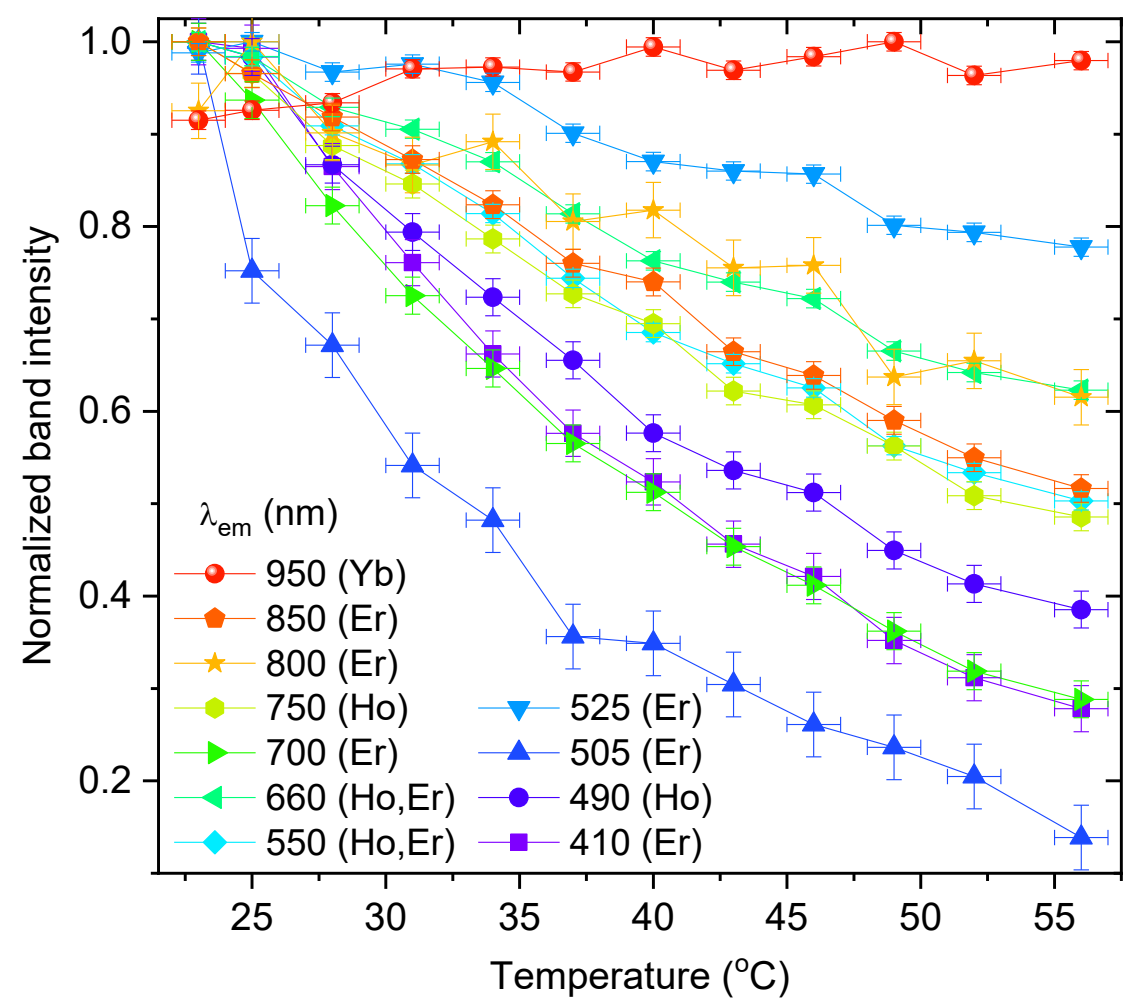

Figure S5. Normalized, integrated luminescence intensity of the emission bands (below excitation wavelength) as a function of temperature, for the $\mathrm{Sr}_{2} \mathrm{LuF}_{7}: \mathrm{Yb}^{3+}-\mathrm{Ho}^{3+}-\mathrm{Er}^{3+} \mathrm{NPs}\left(\lambda_{\mathrm{ex}}=975 \mathrm{~nm}\right)$.

Table S2. Transitions used for different LIR and lifetime-based thermometric techniques, wavelength, fitted equations, correlation coefficients, maximum $S_{r}$ and the corresponding temperature values.

\begin{tabular}{|c|c|c|c|c|c|}
\hline Transitions & $\lambda(\mathbf{n m})$ & Equation & $R^{2}$ & $\begin{array}{l}S_{r \text { MAX }} \\
\left(\% K^{-1}\right)\end{array}$ & $T\left({ }^{0} \mathrm{C}\right)$ \\
\hline $\begin{array}{l}\left(\mathrm{Yb}^{3+}\right)^{2} \mathrm{~F}_{5 / 2} \rightarrow{ }^{2} \mathrm{~F}_{7 / 2} / \\
\left(\mathrm{Er}^{3+}\right)^{2} \mathrm{H}_{9 / 2} \rightarrow{ }^{4} \mathrm{I}_{15 / 2}\end{array}$ & $950 / 410$ & $\begin{array}{c}1.722 \times 10^{-2} T^{2}- \\
0.3425 T+9.950\end{array}$ & 0.997 & 4.57 & 32 \\
\hline $\begin{array}{l}\left(\mathrm{Yb}^{3+}\right)^{2} \mathrm{~F}_{5 / 2} \rightarrow{ }^{2} \mathrm{~F}_{7 / 2} / \\
\left(\mathrm{Ho}^{3+}\right){ }^{3} \mathrm{~K}_{8},{ }^{5} \mathrm{~F}_{2} \rightarrow{ }^{5} \mathrm{I}_{8}\end{array}$ & $950 / 490$ & $\begin{array}{c}2.14 \times 10^{-3} T^{2}+ \\
9.449 \times 10^{-2} T+1.257\end{array}$ & 0.995 & 4.23 & 23 \\
\hline $\begin{array}{l}\left(\mathrm{Yb}^{3+}\right)^{2} \mathrm{~F}_{5 / 2} \rightarrow{ }^{2} \mathrm{~F}_{7 / 2} / \\
\left(\mathrm{Er}^{3+}\right)^{4} \mathrm{G}_{11 / 2} \rightarrow{ }^{4} \mathrm{I}_{13 / 2}\end{array}$ & $950 / 505$ & $\begin{array}{c}0.1480 T^{2}+ \\
1.6862 T-24.15\end{array}$ & 0.995 & 9.15 & 23 \\
\hline $\begin{array}{l}\left(\mathrm{Yb}^{3+}\right)^{2} \mathrm{~F}_{5 / 2} \rightarrow{ }^{2} \mathrm{~F}_{7 / 2} / \\
\left(\mathrm{Er}^{3+}\right)^{2} \mathrm{H}_{11 / 2} \rightarrow{ }^{4} \mathrm{I}_{15 / 2}\end{array}$ & $950 / 525$ & $\begin{array}{c}-7.944 \times 10^{-5} T^{2}+ \\
3.38 \times 10^{-2} T+1.564\end{array}$ & 0.970 & 1.31 & 23 \\
\hline $\begin{array}{c}\left(\mathrm{Yb}^{3+}\right){ }^{2} \mathrm{~F}_{5 / 2} \rightarrow{ }^{2} \mathrm{~F}_{7 / 2} / \\
\left(\mathrm{Ho}^{3+}\right){ }^{5} \mathrm{~S}_{2},{ }^{5} \mathrm{~F}_{4} \rightarrow{ }^{5} \mathrm{I}_{8} \& \\
\left(\mathrm{Er}^{3+}\right){ }^{4} \mathrm{~S}_{3 / 2} \rightarrow{ }^{4} \mathrm{I}_{15 / 2}\end{array}$ & $950 / 550$ & $\begin{array}{c}2.095 \times 10^{-5} T^{2}+ \\
1.99 \times 10^{-3} T+4.398 \times 10^{-2}\end{array}$ & 0.994 & 2.93 & 23 \\
\hline $\begin{array}{l}\left(\mathrm{Yb}^{3+}\right)^{2} \mathrm{~F}_{5 / 2} \rightarrow{ }^{2} \mathrm{~F}_{7 / 2} / \\
\left(\mathrm{Ho}^{3+}\right){ }^{5} \mathrm{~F}_{5} \rightarrow{ }^{5} \mathrm{I}_{8} \& \\
\left(\mathrm{Er}^{3+}\right){ }^{4} \mathrm{~F}_{9 / 2} \rightarrow{ }^{4} \mathrm{I}_{15 / 2}\end{array}$ & $950 / 660$ & $\begin{array}{c}-7.602 \times 10^{-7} T^{2}+ \\
2.05 \times 10^{-3} T+3.979 \times 10^{-2}\end{array}$ & 0.991 & 2.33 & 23 \\
\hline
\end{tabular}




\begin{tabular}{|c|c|c|c|c|c|}
\hline $\begin{aligned} &\left(\mathrm{Yb}^{3+}\right)^{2} \mathrm{~F}_{5 / 2} \rightarrow{ }^{2} \mathrm{~F}_{7 / 2} / \\
&\left(\mathrm{Er}^{3+}\right){ }^{4} \mathrm{~F}_{7 / 2} \rightarrow{ }^{4} \mathrm{I}_{13 / 2}\end{aligned}$ & $950 / 700$ & $\begin{array}{c}3.84 \times 10^{-3} T^{2}- \\
1.207 \times 10^{-2} T+1.719\end{array}$ & 0.999 & 4.74 & 23 \\
\hline $\begin{array}{l}\left(\mathrm{Yb}^{3+}\right)^{2} \mathrm{~F}_{5 / 2} \rightarrow{ }^{2} \mathrm{~F}_{7 / 2} / \\
\left(\mathrm{Ho}^{3+}\right){ }^{5} \mathrm{~S}_{2},{ }^{5} \mathrm{~F}_{4} \rightarrow{ }^{5} \mathrm{I}_{7}\end{array}$ & $950 / 750$ & $\begin{array}{c}4.990 \times 10^{-4} T^{2}+ \\
4.68 \times 10^{-2} T+0.9623\end{array}$ & 0.999 & 3.03 & 23 \\
\hline $\begin{array}{c}\left(\mathrm{Yb}^{3+}\right){ }^{2} \mathrm{~F}_{5 / 2} \rightarrow{ }^{2} \mathrm{~F}_{7 / 2} / \\
\left(\mathrm{Er}^{3+}\right){ }^{4} \mathrm{I}_{9 / 2} \rightarrow{ }^{4} \mathrm{I}_{15 / 2}\end{array}$ & $950 / 800$ & $\begin{array}{c}4.18 \times 10^{-3} T^{2}+ \\
0.2645 T+21.43\end{array}$ & 0.972 & 1.56 & 33 \\
\hline $\begin{aligned}\left(\mathrm{Yb}^{3+}\right){ }^{2} \mathrm{~F}_{5 / 2} & \rightarrow{ }^{2} \mathrm{~F}_{7 / 2} / \\
\left(\mathrm{Er}^{3+}\right){ }^{4} \mathrm{~S}_{3 / 2} & \rightarrow{ }^{4} \mathrm{I}_{13 / 2}\end{aligned}$ & $950 / 850$ & $\begin{array}{c}3.163 \times 10^{-4} T^{2}+ \\
1.289 \times 10^{-2} T+0.6776\end{array}$ & 0.998 & 2.40 & 23 \\
\hline $\begin{array}{l}\left(\mathrm{Er}^{3+}\right){ }^{4} \mathrm{I}_{13 / 2} \rightarrow{ }^{4} \mathrm{I}_{15 / 2} / \\
\left(\mathrm{Ho}^{3+}\right){ }^{5} \mathrm{I}_{6} \rightarrow{ }^{5} \mathrm{I}_{8}\end{array}$ & $1550 / 1200$ & $\begin{array}{c}1.588 \times 10^{-2} T^{2}- \\
0.3499 T+105.96\end{array}$ & 0.984 & 1.05 & 56 \\
\hline $\begin{array}{l}\left(\mathrm{Er}^{3+}\right)^{4} \mathrm{I}_{13 / 2} \rightarrow{ }^{4} \mathrm{I}_{15 / 2} / \\
\left(\mathrm{Yb}^{3+}\right){ }^{2} \mathrm{~F}_{5 / 2} \rightarrow{ }^{2} \mathrm{~F}_{7 / 2}\end{array}$ & $1550 / 1000$ & $\begin{array}{c}1.797 \times 10^{-4} T^{2}+ \\
7.11 \times 10^{-3} T+1.147\end{array}$ & 0.989 & 1.29 & 56 \\
\hline $\begin{aligned}\left(\mathrm{Ho}^{3+}\right){ }^{5} \mathrm{I}_{6} & \rightarrow{ }^{5} \mathrm{I}_{8} / \\
\left(\mathrm{Yb}^{3+}\right){ }^{2} \mathrm{~F}_{5 / 2} & \rightarrow{ }^{2} \mathrm{~F}_{7 / 2}\end{aligned}$ & $1200 / 1000$ & $\begin{array}{c}-2.332 \times 10^{-7} T^{2}+ \\
9.462 \times 10^{-5} T+1.12 \times 10^{-2}\end{array}$ & 0.963 & 0.63 & 23 \\
\hline $\begin{array}{c}\left(\mathrm{Er}^{3+}\right){ }^{4} \mathrm{~S}_{3 / 2} \rightarrow{ }^{4} \mathrm{I}_{13 / 2} \\
\text { (lifetime) }\end{array}$ & 850 & $-0.1753 T+82.41$ & 0.997 & 0.24 & 56 \\
\hline
\end{tabular}

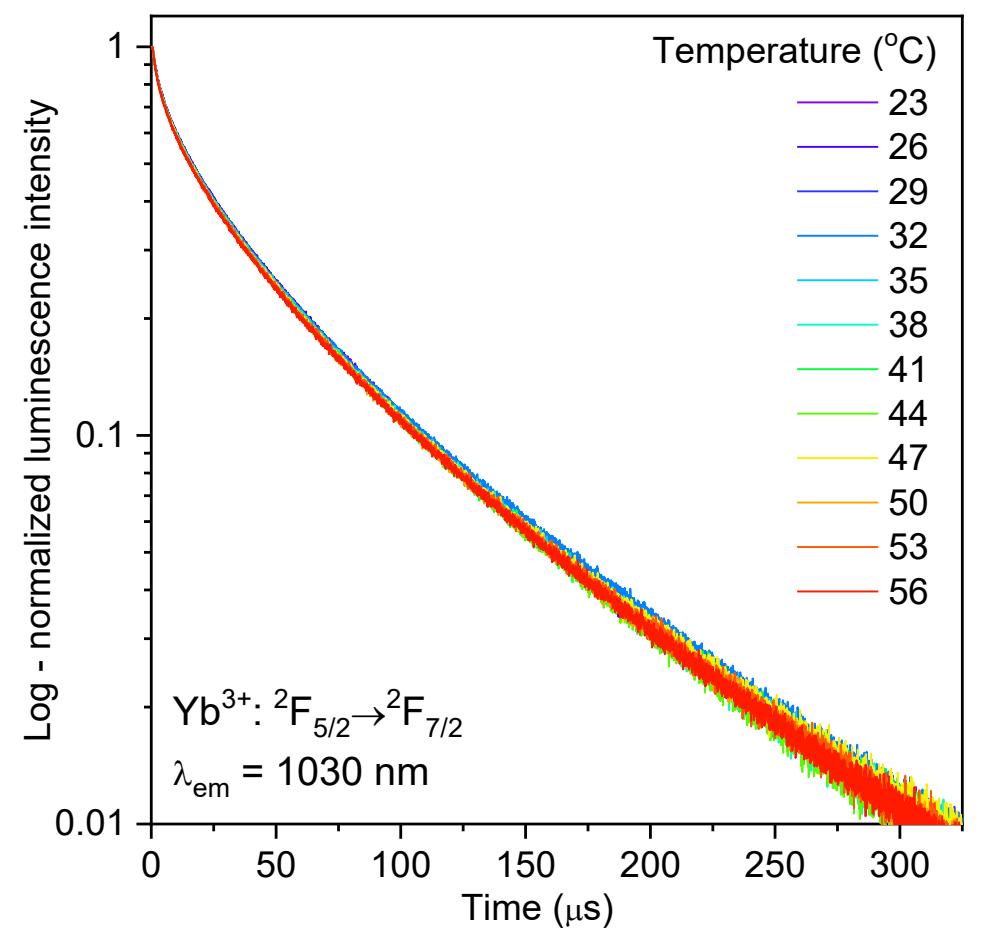

Figure S6. Luminescence decay curves of $\mathrm{Yb}^{3+}\left({ }^{2} \mathrm{~F}_{5 / 2} \rightarrow{ }^{2} \mathrm{~F}_{7 / 2}\right)$ measured at different temperature values (23-56 $\left.{ }^{\circ} \mathrm{C}\right)$, for the $\mathrm{Sr}_{2} \mathrm{LuF}_{7}: \mathrm{Yb}^{3+}-\mathrm{Ho}^{3+}-\mathrm{Er}^{3+} \mathrm{NPs} ; \lambda_{\mathrm{ex}}=975 \mathrm{~nm}$. 


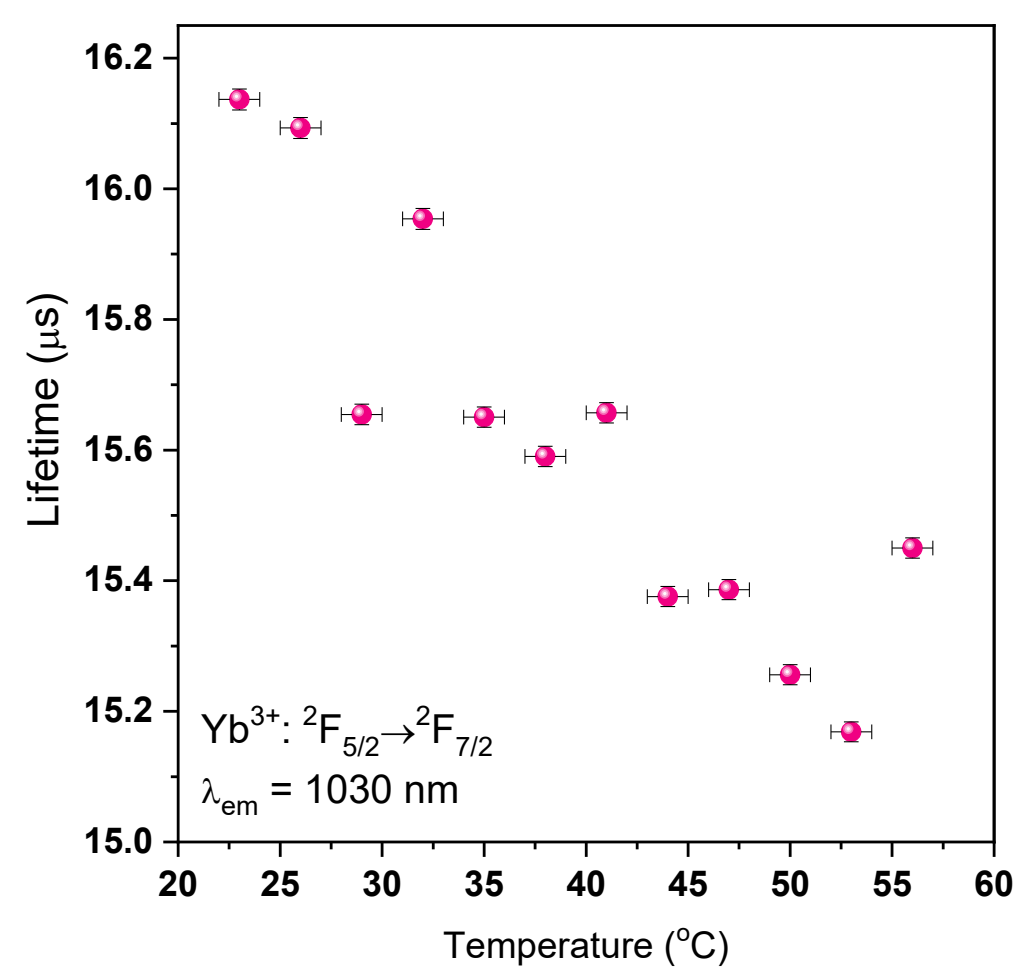

Figure S7. Determined luminescence lifetimes of $\mathrm{Yb}^{3+}\left({ }^{2} \mathrm{~F}_{5 / 2} \rightarrow{ }^{2} \mathrm{~F}_{7 / 2}\right)$ as a function of temperature, for the $\mathrm{Sr}_{2} \mathrm{LuF}_{7}: \mathrm{Yb}^{3+}-\mathrm{Ho}^{3+}-\mathrm{Er}^{3+} \mathrm{NPs} ; \lambda_{\mathrm{ex}}=975 \mathrm{~nm}$.

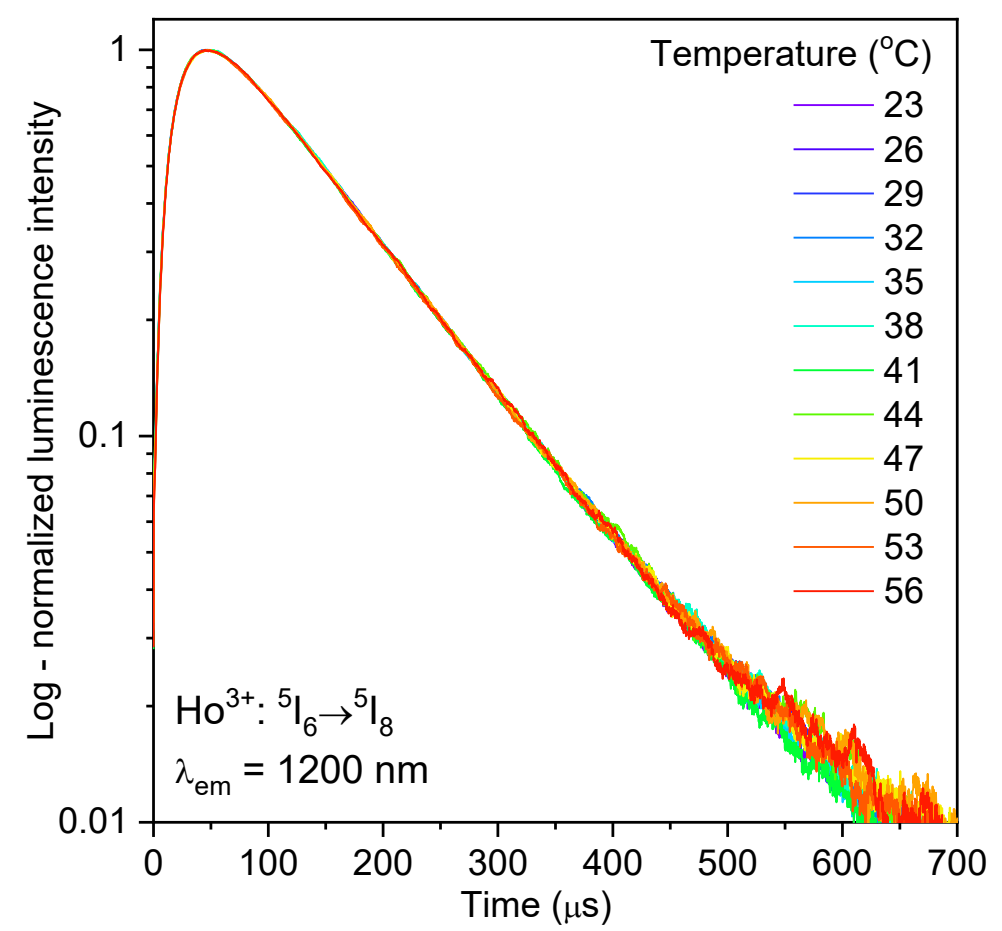

Figure S8. Luminescence decay curves of $\mathrm{Ho}^{3+}\left({ }^{5} \mathrm{I}_{6} \rightarrow{ }^{5} \mathrm{I}_{8}\right)$ measured at different temperature values $\left(23-56^{\circ} \mathrm{C}\right)$, for the $\mathrm{Sr}_{2} \mathrm{LuF}_{7}: \mathrm{Yb}^{3+}-\mathrm{Ho}^{3+}-\mathrm{Er}^{3+} \mathrm{NPs} ; \lambda_{\mathrm{ex}}=975 \mathrm{~nm}$. 


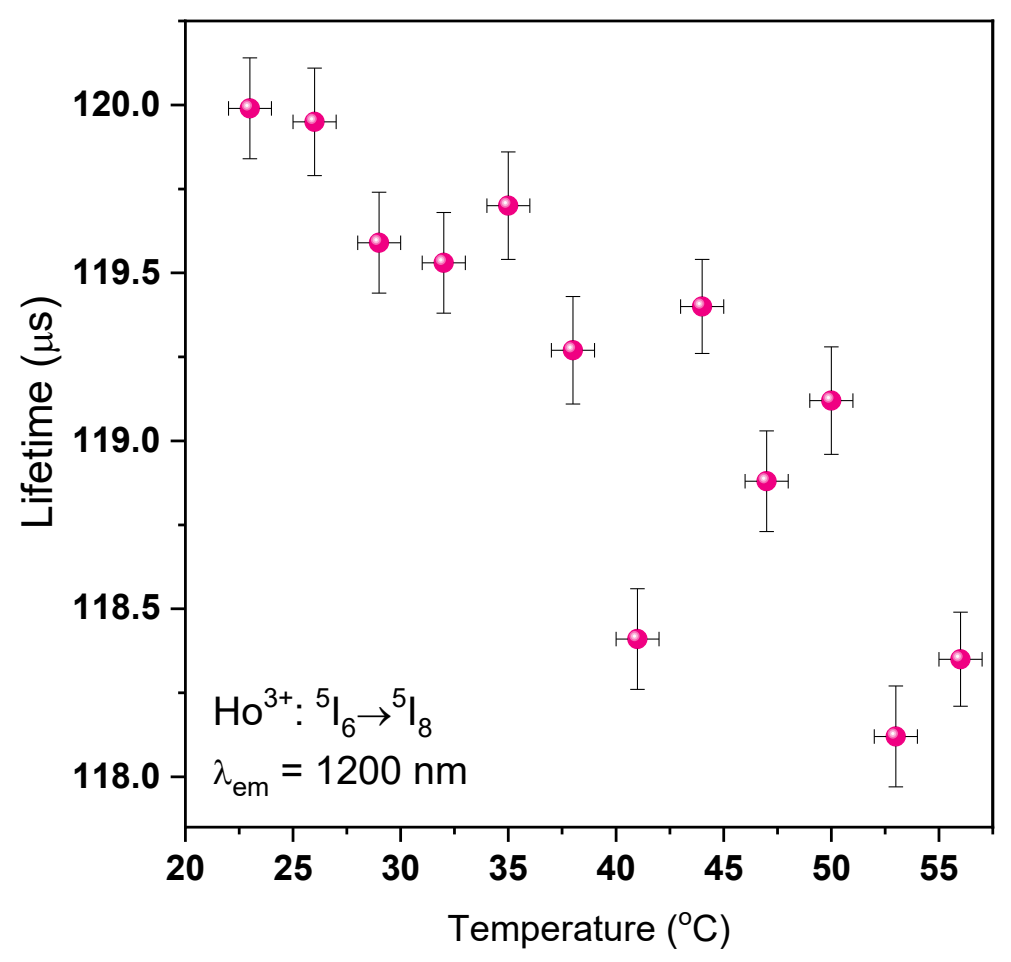

Figure S9. Determined luminescence lifetimes of $\mathrm{Ho}^{3+}\left({ }^{5} \mathrm{I}_{6} \rightarrow{ }^{5} \mathrm{I}_{8}\right)$ as a function of temperature, for the $\mathrm{Sr}_{2} \mathrm{LuF}_{7}: \mathrm{Yb}^{3+}-\mathrm{Ho}^{3+}-\mathrm{Er}^{3+} \mathrm{NPs} ; \lambda_{\mathrm{ex}}=975 \mathrm{~nm}$.

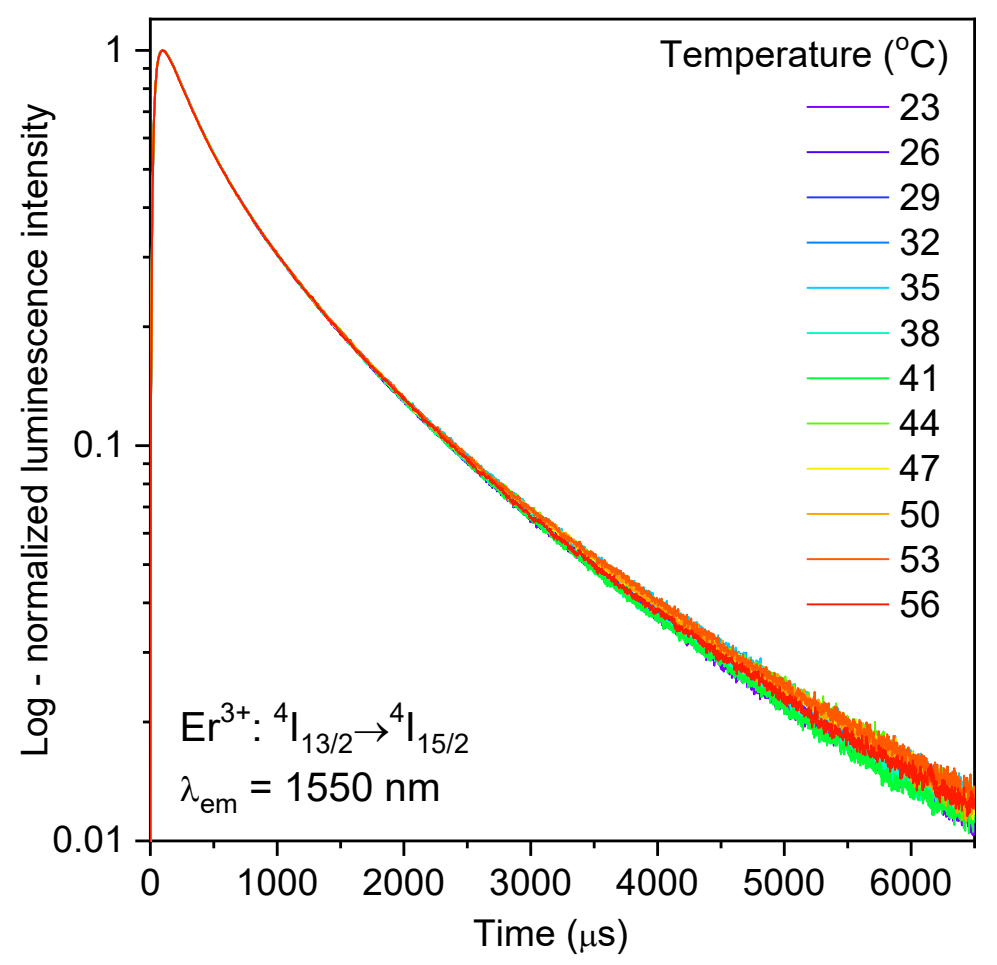

Figure S10. Luminescence decay curves of $\operatorname{Er}^{3+}\left({ }^{4} \mathrm{I}_{13 / 2} \rightarrow{ }^{4} \mathrm{I}_{15 / 2}\right)$ measured at different temperature values $\left(23-56^{\circ} \mathrm{C}\right)$, for the $\mathrm{Sr}_{2} \mathrm{LuF}_{7}: \mathrm{Yb}^{3+}-\mathrm{Ho}^{3+}-\mathrm{Er}^{3+} \mathrm{NPs} ; \lambda_{\mathrm{ex}}=975 \mathrm{~nm}$. 


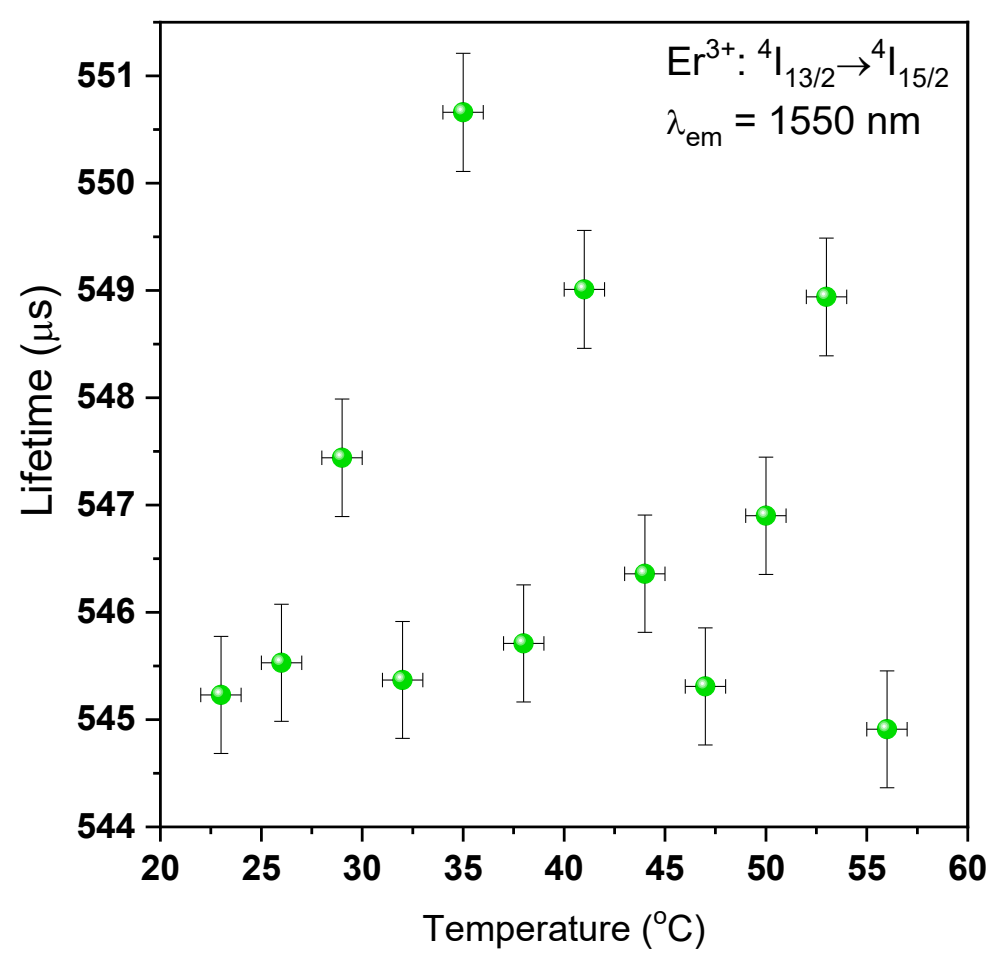

Figure S11. Determined luminescence lifetimes of $\operatorname{Er}^{3+}\left({ }^{4} \mathrm{I}_{13 / 2} \rightarrow{ }^{4} \mathrm{I}_{15 / 2}\right)$ as a function of temperature, for the $\mathrm{Sr}_{2} \mathrm{LuF}_{7}: \mathrm{Yb}^{3+}-\mathrm{Ho}^{3+}-\mathrm{Er}^{3+} \mathrm{NPs} ; \lambda_{\mathrm{ex}}=975 \mathrm{~nm}$. 

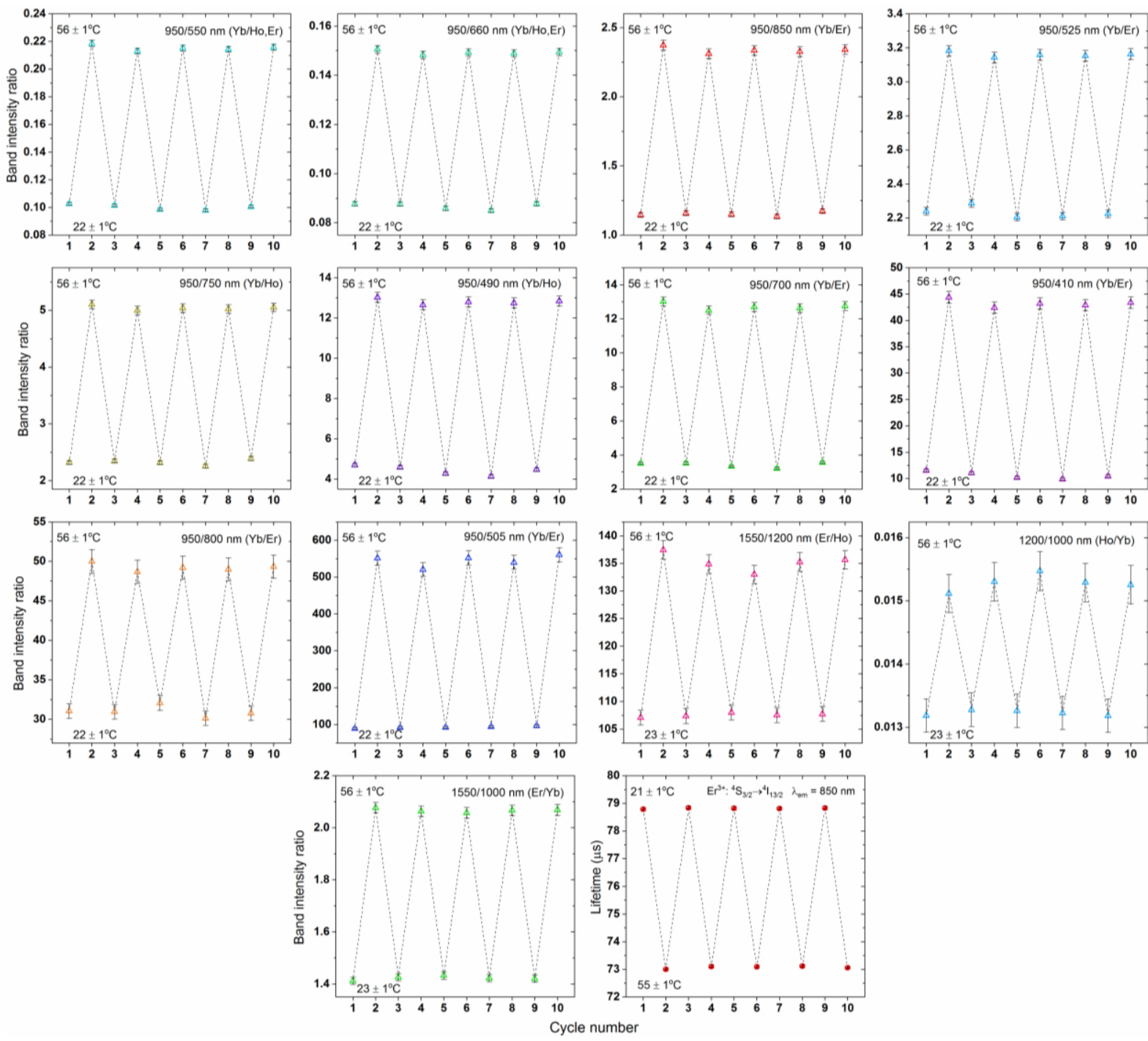

Figure S12. Repetitive measurements of the measured parameter - MP (band ratios - LIR or lifetime), by cycling the sample between ambient and elevated temperature; $\lambda_{\mathrm{ex}}=975 \mathrm{~nm}$. 

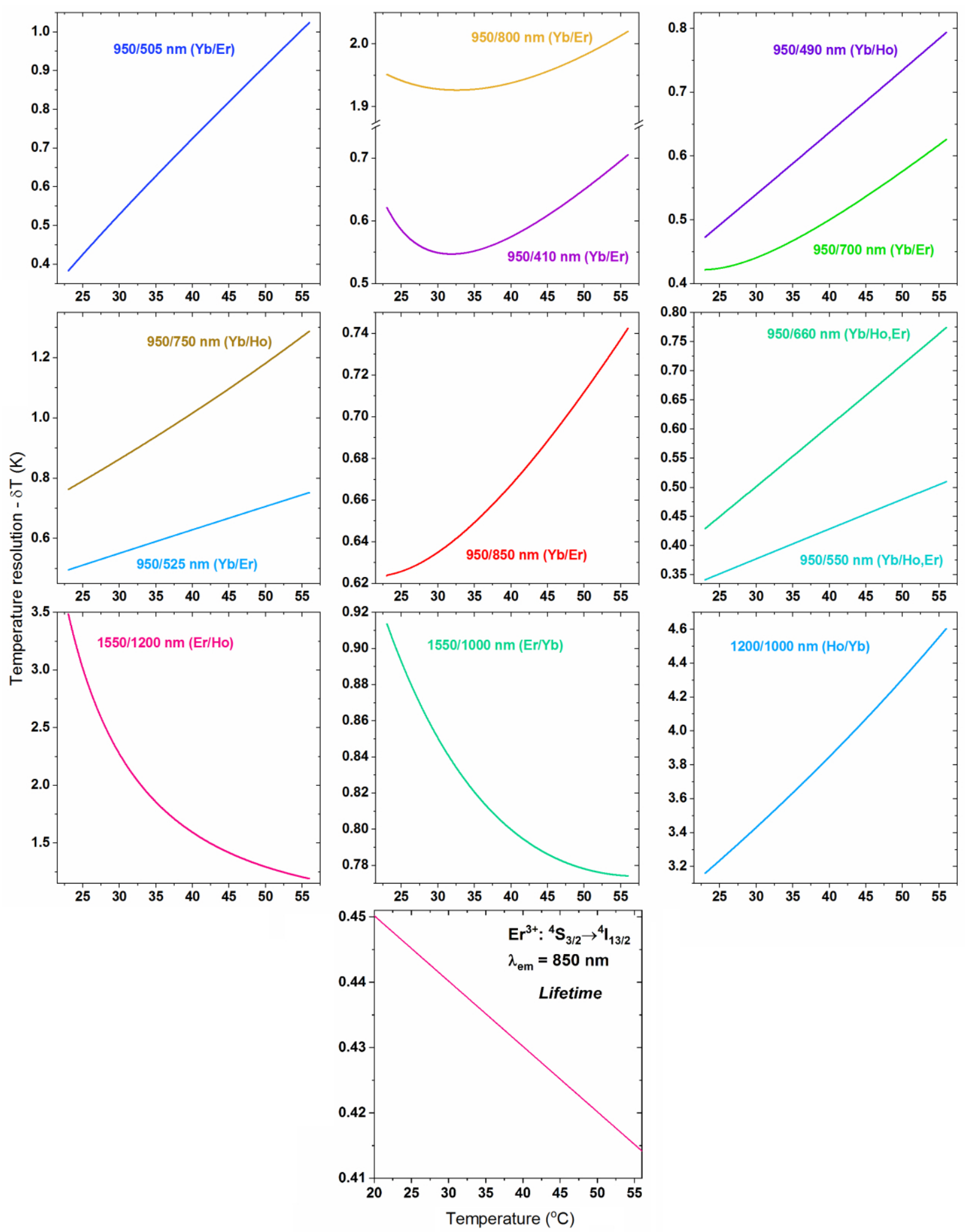

Figure S13. Temperature sensing resolution (uncertainty; $\delta T$ ) as a function of temperature, for the $\mathrm{Sr}_{2} \mathrm{LuF}_{7}: \mathrm{Yb}^{3+}-\mathrm{Ho}^{3+}-\mathrm{Er}^{3+} \mathrm{NPs}\left(\lambda_{\mathrm{ex}}=975 \mathrm{~nm}\right)$, for ratio-metric technique and lifetime-based sensing (bottom). 


\section{References:}

[1] T. Grzyb, D. Przybylska, Formation Mechanism, Structural, and Upconversion Properties of Alkaline Rare-Earth Fluoride Nanocrystals Doped With Yb 3+ /Er 3+ Ions, Inorg. Chem. 57 (2018) 6410-6420. doi:10.1021/acs.inorgchem.8b00484.

[2] M. Pollnau, D.R. Gamelin, S.R. Lüthi, H.U. Güdel, M.P. Hehlen, Power dependence of upconversion luminescence in lanthanide and transition-metal-ion systems, Phys. Rev. B. 61 (2000) 3337-3346. doi:10.1103/PhysRevB.61.3337.

[3] Y. Lei, H. Song, L. Yang, L. Yu, Z. Liu, G. Pan, et al., Upconversion luminescence, intensity saturation effect, and thermal effect in Gd2O3:Er3,Yb3+ nanowires, J. Chem. Phys. 123 (2005) 174710. doi:10.1063/1.2087487. 